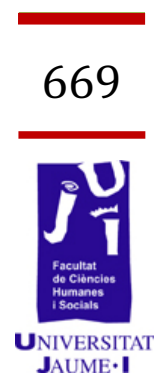

\title{
La interferencia de la LM en el discurso de profesores brasileños de español como lengua extranjera
}


Algunos autores como Almeida Filho (1995) afirman que entre las lenguas románicas el portugués y el español son las que tienen más afinidad. En consecuencia, en razón de la similitud (morfológica, sintáctica, semántica y fonético fonológica), no existen alumnos luso hablantes considerados como "principiantes" en español, visto que normalmente ya tienen adquirida la capacidad de comprender parte del idioma, tanto en el registro hablado como en el escrito. Por otro lado y paradójicamente, una de las mayores dificultades de dichos estudiantes es superar las similitudes existentes en los dos idiomas, que terminan por facilitar las interferencias de la lengua materna en la extranjera. No siempre es fácil, aún en niveles muy altos de conocimiento de la lengua extranjera, desprenderse de algunos aspectos propios de la lengua materna. En el caso de los docentes de español hay que añadir la preocupación por la influencia que las interferencias puedan ejercer en el aprendizaje del alumno. Por esa razón, este estudio pretende averiguar si en el discurso oral de los profesores brasileños de español como lengua extranjera, se presentan signos de interferencia de la lengua materna. La colecta de datos se hará con base en la grabación de clases y entrevistas en audio y cuestionario escrito. Se delimitarán los análisis en el marco de las subcompetencias gramatical y léxico-semántica y se usará el método de análisis de errores apoyado en la lingüística contrastiva y la lingüística de corpus. Su carácter es cuantitativo y cualitativo, buscando, por un lado estudiar la frecuencia de los errores y, por otro, describir y detallar los diferentes tipos con base en la gramática normativa.

Palabras clave: interferencia, análisis contrastivo, análisis de errores, lengua española, lengua portuguesa

\section{Introducción}

La presente investigación es parte de una tesis doctoral en proceso de desarrollo, fruto de un convenio de cotutela entre la Universidad Jaume I y la Universidade Federal de Santa Catarina en Brasil. De esta manera, el objetivo de este artículo es presentar el trabajo que está en desarrollo y, por ello, no se presentarán resultados ni conclusiones, sino se intentará discutir el proceso de la investigación, sus objetivos, métodos y la población de estudio.

En el contexto de aprendizaje de una lengua extranjera, no podemos ignorar la influencia de la lengua materna, tan ampliamente discutida en las últimas décadas. En el caso de lenguas muy parecidas, como el portugués y el español, sabemos que no siempre la similitud es un factor facilitador del aprendizaje.

Mucho se ha estudiado sobre las transferencias encontradas en la interlengua de los aprendientes aunque con denominaciones diferentes, como interferencia (Weinreich, 1953), Crosslinguistic influence, 
(Kellerman, 1995), borrowing (Corder, 1981), bidirectional transfer (Ellis, 1994), entre otros, pero aun queda un largo camino por percorrer cuando nos referimos a los rasgos de la lengua materna persistentes en aquellos individuos considerados "avanzados" dentro del marco global de competencia lingüística.

Durante la práctica docente como profesora de español como lengua extranjera en mi país de origen (Brasil), y también compartiendo experiencia con mis compañeros de profesión en los más diferentes ámbitos, pude entender que, no siempre es fácil, aún en niveles muy altos de proficiencia, desprenderse de algunos aspectos propios de la lengua materna que merecerían un estudio detallado.

Es relativamente común encontrar en la bibliografía internacional especializada, diversos trabajos sobre la interferencia de la lengua materna en el aprendizaje de una lengua extranjera. En países con un histórico muy reciente en la investigación de la lingüística aplicada como Brasil, en los últimos años, se empiza a notar el creciente interés por el tema, aunque exista mucho camino por recorrer, principalemente cuando nos referimos a aprendices/hablantes de niveles más avanzados.

Si añadimos a esta realidad, que el país pasa por una época histórica en relación a la enseñanza de ELE, establecida por la Ley 11.161 de 5 de Agosto de $2005^{1}$, que obliga a todos los centros de enseñanza media a ofrecer el español como segunda lengua, podemos hablar de una producción "en masa" de profesores en los últimos años. Según los últimos datos proporcionados por el Instituto Nacional de Investigación Brasileño $(2009)^{2}$, sólo un $40 \%$ de los profesores de lengua extranjera del país tienen formación específica a nivel superior. En aquel mismo año, según el Ministerio de Educación, el país contaba tan solo con 6.000 profesores en Enseñanza Media, apenas un $20 \%$ del total necesario para cumplir la ley. Esto explica la creciente oferta de plazas para profesores universitarios por todo el país. La urgencia por formar profesores es tan evidente como la necesidad de formación del cuerpo docente existente.

Por estas razones creemos que sean necesarias más investigaciones que determinen cuál es la verdadera realidad lingüística de los profesores de español en el país y las posibles implicaciones para los alumnos en caso de que estén recibiendo un input inadecuado.

\section{Objetivos}

Los objetivos de esta investigación son:

\subsection{Objetivos generales:}

Averiguar si en el discurso oral de la población estudiada (profesores brasileños de español como lengua extranjera), se presentan signos de interferencia de la lengua materna, identificarlos y explicarlos, así como

\footnotetext{
${ }^{1}$ Disponible en línea: http://www.planalto.gov.br/ccivil_03/_Ato2004-2006/2005/Lei/L11161.htm [02/01/2015]

2 Disponible en línea: http://www.inep.gov.br/download/censo/2009/Estudo_Professor_1.pdf [02/01/2015]
} 
reconocer qué tipos son más frecuentes. Se delimitarán los análisis en el marco de las subcompetencias, gramatical y léxico-semántica.

\subsection{Objetivos específicos:}

a) Describir y clasificar los errores gramaticales y descriptivos (Corder 1981) de interferencia;

b) Verificar si hay otros tipos de errores y si los de transferencia son predominantes;

c) Verificar cuáles son los errores de interferencia gramatical más frecuentes;

d) Verificar si coinciden los tipos de errores de interferencia gramatical y descriptivo entre las profesoras;

e) Verificar si coincide la frecuencia de los errores de interferencia gramatical y descriptivo entre las profesoras;

f) Verificar si el nivel de instrucción influye en la cantidad de las interferencias;

g) Verificar si los tipos de errores gramatical y descriptivo y su frecuencia varían según el contexto de colecta del corpus (clases x entrevista) como sostiene Tarone (1992).

\section{Material y método}

\subsection{Métodos}

En esta investigación se usa el método de análisis de errores y el análisis contrastivo, puesto que entendemos que no son antagónicos sino complementarios. El carácter del estudio es cuantitativo y cualitativo, buscando, por un lado estudiar la frecuencia de los errores y por otro describir y detallar los diferentes tipos con base en la gramática. También se recurrirá a la gramática contrastiva, puesto que constituye la manera más clara y precisa de comparar y explicar los errores de interferencia.

El método inductivo también se hace presente, puesto que se observarán y se analizarán los hechos, se clasificarán los errores, partiendo del estudio detallado de casos particulares para llegar a la generalización. Se usarán los conocimientos de lengua materna de la investigadora para clasificar los errores en un primero momento, para luego contrastar las gramáticas.

La periodicidad de la recogida de datos caracteriza este estudio como sincrónico o transversal. Los datos se recolectaron de tres maneras distintas, todas durante el mes de septiembre de 2013: a) grabaciones de audio una o más clases de 50 minutos y b) grabaciones de audio de una entrevista semiestructurada de aproximadamente 30 minutos c) test objetivo de gramática.

\subsection{Etapas}

De acuerdo con el método de análisis de errores que rige este trabajo, las etapas de la investigación son:

1. Compilación del corpus de datos;

2. Identificación de los errores; 
3. Descripción de los errores;

4. Clasificación de acuerdo con las taxonomías;

5. Explicación de los errores;

6. Discusión de los resultados;

7. Implicaciones didácticas para el proceso de enseñanzaaprendizaje.

En el momento de la redacción de este artículo se ha cumplido con las dos primeras etapas referidas arriba.

\subsection{Población}

La población estudiada constituye la totalidad del cuerpo docente de español como lengua extranjera en centros de enseñanza reglada en la ciudad dónde se recopilaron los datos. Todos los individuos son naturales de Brasil, de la región sur del país, de edades entre 29 y 51 años, del sexo femenino y han cursado el la misma carrera en la misma universidad. La mayoría ( 8 de 10) ha aprendido el español como lengua extranjera en la misma universidad, puesto que no se exige ningun nivel de lengua para acceder a los estudios universitarios.

Los docentes estudiados son naturales de las localidades de ljuí y Panambi, pertenecientes a la región noroeste del estado de Rio Grande do Sul dónde residen y trabajan. Una única docente trabaja en una tercera ciudad de la región central y la capital del país: Brasilia. Los centros en que imparten clase son de educación primaria y secundaria, privados 0 públicos, para diferentes grupos que equivaldrían a los de la ESO y del Bachiller de la enseñanza española.

El número total de sujetos participantes es de diez profesoras y la mayoría no tenía ningún conocimiento o conocimientos muy básicos al empezar la carrera ${ }^{3}$. Mayores detalles se pueden observar en las tablas abajo.

\footnotetext{
3 Las carreras para profesores en Brasil se llaman «Licenciaturas». Consisten en cursos de grado con duración media de cuatro años que forman docentes en las más diferentes áreas. La carrera que estudió la población de este estudio, se llama "Licenciatura em Língua Extrangeira: Espanhol», grado que no forma filólogos, sino profesores de la lengua con formación en didáctica, los cuales terminan la carrera con el diploma correspondiente a profesor de español como lengua extranjera.
} 
Tabla 1. Población

\begin{tabular}{|c|c|c|c|c|c|c|c|}
\hline $\begin{array}{c}\text { Sujet } \\
\text { o }\end{array}$ & $\begin{array}{c}\text { Eda } \\
\text { d }\end{array}$ & $\begin{array}{c}\text { Tipo de } \\
\text { institució } \\
\text { n en que } \\
\text { trabaja }\end{array}$ & $\begin{array}{l}\text { Grupos } \\
\text { a los } \\
\text { cuales } \\
\text { impart } \\
\text { e clases }\end{array}$ & Formación & $\begin{array}{l}\text { Formación reglada } \\
\text { adicional en Brasil }\end{array}$ & $\begin{array}{l}\text { Formació } \\
n \\
\text { adicional } \\
\text { en el } \\
\text { extranjer } \\
0\end{array}$ & $\begin{array}{l}\text { Tiempo } \\
\text { de } \\
\text { profesió } \\
\text { n en } \\
\text { años }\end{array}$ \\
\hline 1 & 29 & pública & $\begin{array}{c}10,2 \text { y } \\
\text { 3o año } \\
\mathrm{EM}^{4}\end{array}$ & $\begin{array}{l}\text { Licenciatura } \\
5 \text { en Español } \\
\text { Lengua } \\
\text { Extranjera }\end{array}$ & $\begin{array}{l}\text { Posgrado en } \\
\text { metodología de la } \\
\text { enseñanza de } \\
\text { portugués y lengua } \\
\text { extranjera }\end{array}$ & no & 2 \\
\hline 2 & 47 & pública & $\begin{array}{l}\text { 6o año } \\
\text { EB }{ }^{6} \text { a } \\
\text { 3 año } \\
\text { EM }\end{array}$ & $\begin{array}{l}\text { Licenciatura } \\
\text { en Español } \\
\text { Lengua } \\
\text { Extranjera }\end{array}$ & no & no & 14 \\
\hline 3 & 33 & pública & $\begin{array}{c}70,80 \text { y } \\
90 \text { años } \\
\text { EB }\end{array}$ & $\begin{array}{c}\text { Licenciatura } \\
\text { en Español } \\
\text { Lengua } \\
\text { Extranjera }\end{array}$ & $\begin{array}{c}\text { Posgrado en } \\
\text { enseñanza de } \\
\text { lenguas extranjeras } \\
\text { - español. } \\
\text { Cursando posgrado } \\
\text { en gestión escolar }\end{array}$ & no & 8 \\
\hline 4 & 30 & pública & $\begin{array}{l}\text { 6으 año } \\
\text { EB a } 30 \\
\text { año EM }\end{array}$ & $\begin{array}{l}\text { Licenciatura } \\
\text { en Español } \\
\text { Lengua } \\
\text { Extranjera }\end{array}$ & no & no & 8 \\
\hline 5 & 30 & privada & $\begin{array}{c}\text { 7으, 8ㅇy y } \\
\text { 9o años } \\
\text { EB }\end{array}$ & $\begin{array}{l}\text { Licenciatura } \\
\text { en Español } \\
\text { Lengua } \\
\text { Extranjera }\end{array}$ & $\begin{array}{c}\text { Cursando posgrado } \\
\text { en } \\
\text { neuropsicopedagogí } \\
\text { a }\end{array}$ & no & 5 \\
\hline 6 & 31 & privada & $\begin{array}{l}\text { 9o año } \\
\text { EB y } 1 \text { o } \\
\text { año EM }\end{array}$ & $\begin{array}{l}\text { Licenciatura } \\
\text { en Español } \\
\text { Lengua } \\
\text { Extranjera }\end{array}$ & no & no & 1 \\
\hline 7 & 34 & privada & $\begin{array}{c}1 \text { 으, } 2 \text { ㅇ y } \\
\text { 3ㅇ año } \\
\text { EM }\end{array}$ & $\begin{array}{l}\text { Licenciatura } \\
\text { en Español } \\
\text { Lengua } \\
\text { Extranjera }\end{array}$ & $\begin{array}{c}\text { Posgrado en psicope } \\
\text { dagogía }\end{array}$ & no & 11 \\
\hline 8 & 29 & pública & $\begin{array}{l}\text { 6ㅇ año } \\
\text { EB a 3ㅇ } \\
\text { año EM }\end{array}$ & $\begin{array}{l}\text { Licenciatura } \\
\text { en Español } \\
\text { Lengua } \\
\text { Extranjera }\end{array}$ & no & no & 8 \\
\hline
\end{tabular}

\footnotetext{
${ }^{4}$ Enseñanza Media brasileña.

${ }^{5}$ Traducción libre de la autora. Carrera que prepara docentes de español como lengua extranjera, explicada en la referencia número 3.

${ }^{6}$ Enseñanza Básica brasileña.
} 


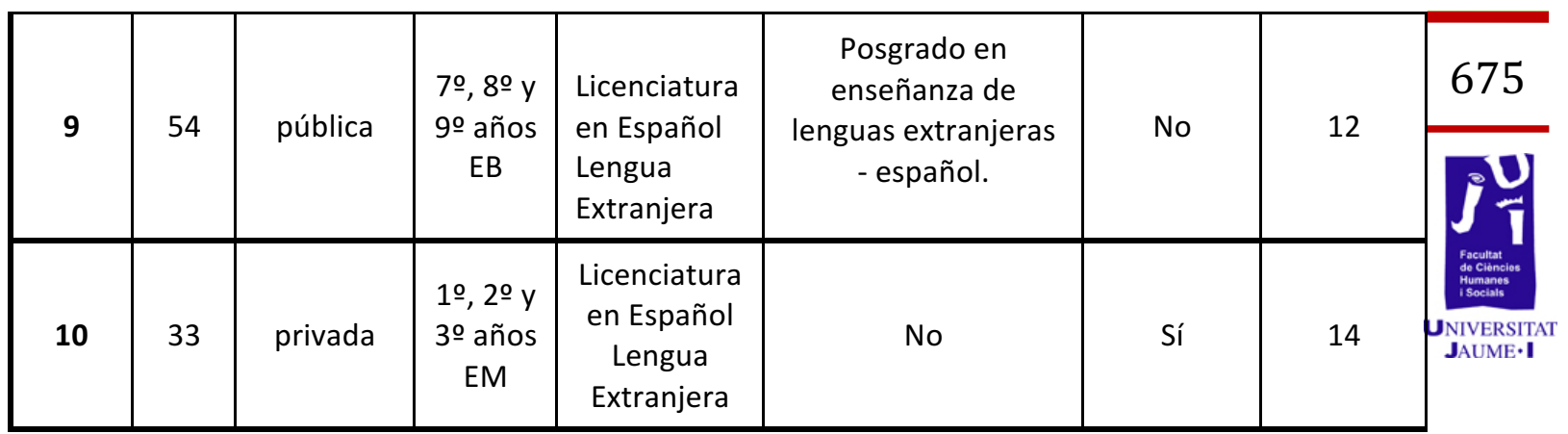

Tabla 2. Conocimiento de la LE al empezar la carrera

\begin{tabular}{|c|c|}
\hline Sujeto & $\begin{array}{c}\text { Nivel de conocimiento de lengua española antes de empezar la } \\
\text { carrera }\end{array}$ \\
\hline 1 & básico \\
\hline 2 & medio \\
\hline 3 & ninguno \\
\hline 4 & básico \\
\hline 5 & ninguno \\
\hline 6 & básico \\
\hline 7 & medio \\
\hline 8 & ninguno \\
\hline 9 & medio \\
\hline 10 & ninguno \\
\hline
\end{tabular}

\subsection{Técnicas e instrumentos de recolección de datos}

La recolección del corpus se realizaró en tres partes distintas y complementarias: primero, la grabación de audio de las clases que imparten normalmente los profesores, en el grupo de su elección, con una duración de una hora de clase mínimo, que corresponde a 50 minutos.

Las grabaciones se realizaron con la supervisión de la investigadora, aunque se haya eximido su presencia en clase con el objetivo de evitar alteraciones en los resultados motivadas por un incremento en el nivel de ansiedad del los profesores y alumnos.

En segundo lugar, se realizaró una entrevista guiada de unos 30 minutos aproximadamente, también grabada en audio. Recoger dos tipos de muestras en situaciones comunicativas distintas puede proporcionarnos un panorama más completo de la competencia lingüística y comunicativa de los sujetos, así como atender a la posible variabilidad que pueden tener las locuciones según el contexto de recogida de datos, como sostiene Tarone (1992). La entrevista tuvo como pauta la realización de preguntas que nos proporcionara un mayor grado de información 
personal y profesional de las profesoras. Esto nos permitirá trazar el perfil sociológico de cada una, datos relevantes para los objetivos de esta investigación.

Por último, se aplicó un breve cuestionario escrito, tipo test, de juicio gramatical. El objetivo del test es comparar la consciencia de error gramatical que puedan presentar en relación con los errores encontrados en la transcripción de sus grabaciones. De esta manera se pretende entender hasta qué punto son conscientes de los errores que cometen.

Después de la recopilación del material se procededió a la transcripción de las clases y entrevistas, así como a la identificación de los errores. Posteriormente se analizarán las respuestas del test.

\subsection{Identificación, descripción y clasificación de los errores:}

Según los criterios usados para el análisis y categorización de los errores de este estudio, usaremos tres clasificaciones distintas y complementarias:

\subsubsection{Criterio gramatical}

La primera categorización es de criterio gramatical e incluye los niveles sintáctico y léxico-semántico de la gramática normativa.

\subsubsection{Criterio de nivel descriptivo}

La segunda clasificación, usada por Corder (1981: 36), hace referencia al carácter del error en un nivel descriptivo: errores de omisión, adición, selección y orden. El primer tipo, el error de omisión, se observa cuando algún elemento es suprimido en la sentencia. Los errores de adición ocurren cuando alguna unidad gramatical es añadida al enunciado pero en un sitio inapropiado. El tercer tipo de error, de selección, ocurre cuando un elemento no es adecuadamente elegido; y, por último, los errores de orden suceden cuando los elementos presentados son correctos, pero están erróneamente secuenciados. Todos los tipos de errores presentados por Corder se hallan clasificados dentro del nivel lingüístico en el que sean cometidos (fonético, gramático o léxico semántico).

Tabla 2. Tipos de Errores (Corder, 1981)

\begin{tabular}{|c|c|c|c|}
\hline & Fonético & Gramático & Léxico-semántico \\
\hline Omisión & & & \\
\hline Adición & & & \\
\hline Elección & & & \\
\hline Orden & & & \\
\hline
\end{tabular}

Para este estudio, nos proponemos analizar el error a niveles gramático y léxico-semánticos, ya que los de nivel fonético no corresponden a nuestros objetivos. 
La segunda clasificación empleada también alude a la aceptabilidad y adecuación de una oración (Corder, 1981). Según la teoría, una oración puede estar muy bien formada gramaticalmente pero aun así ser totalmente inadecuada para el entorno. De ahí la importancia de entender el contexto al tratar el corpus de errores en las investigaciones.

Una oración aceptable seria aquella que un hablante nativo usa en determinada situación y que es reconocida por otro hablante nativo como perteneciente a su lengua. La capacidad de producir tales sentencias dependería de la competencia del hablante. La adecuación de un tema en su contexto, al contrario de la aceptabilidad, tiene lugar en la actuación, ya que tiene que ver con el uso que se hace en el contexto. Veamos el ejemplo en el que el capataz le dice al jefe (Fernández López, 1995: 213):

(i) Oye, súbeme el sueldo

La frase es correcta gramaticalmente, pero es absolutamente inadecuada al contexto. Por ello, podríamos clasificarla en el ejemplo número dos de la tabla de abajo, propuesta por Corder (1981: 41). Aún podría haber casos de inaceptabilidad, adecuación y algún tipo de error, o bien, inaceptabilidad, inadecuación y aun así figurar error. El único caso exento de error sería el número uno, dónde se tiene aceptabilidad y adecuación:

Tabla 3. Aceptabilidad y adecuación (Corder, 1981)

\begin{tabular}{|c|l|l|l|}
\hline 1 & Aceptable & Adecuado & Libre de error \\
\hline 2 & Aceptable & Inadecuado & Erróneo \\
\hline 3 & Inaceptable & Adecuado & Erróneo \\
\hline 4 & Inaceptable & Inadecuado & Erróneo \\
\hline
\end{tabular}

\subsubsection{Criterio de frecuencia}

La tercera y última clasificación que usaremos en nuestro estudio hace referencia a la frecuencia del error (Fernández López, 1995). De acuerdo con esta tipología, los errores pueden ser: sistemáticos, eventuales, fosilizables, individuales y colectivos. Los errores sistemáticos tienen como característica principal la reincidencia en un determinado periodo, y presentan algún tipo de regularidad acentuada como parte de la interlengua de esta fase.

Los errores eventuales, como el mismo nombre sugiere, se caracterizan por aparecer y desaparecer en un determinado estadio, y, al contrario de los sistemáticos, no necesariamente presentan regularidad. Son errores de desarrollo y tienden a desaparecer con la superación de la etapa de aprendizaje. 
Los fosilizables son aquellos que se repiten en varios estadios sucesivos por ofrecer una mayor resistencia al aprendizaje. Los motivos son difícilmente identificables, podrían darse por varias razones, como, por ejemplo, la complejidad de la estructura, la interferencia de otra lengua, un mal entendimiento de las reglas lingüísticas, la falta de atención, etc. Para Fernandez López, (1995: 212), «en este caso la acción didáctica debe llevar al aprendiz a ser consciente del problema, para ponerlo en situación de analizar la causa y reorganizar sus hipótesis».

Los errores fosilizados son aquellos permanentes y de difícil rectificación. Pueden aparecer involuntariamente en momentos de cansancio o estrés, y suelen ser espontáneos e incontrolables. También pueden aparecer con más constancia en los grupos de hablantes de una lengua extranjera que se integran poco en la sociedad y que pueden llegar a construir su propia norma de la lengua. Para la autora estos errores no parecen corregibles y la mejor actitud es la tolerancia.

Por último, los errores colectivos, son un grupo de errores comunes, frecuentemente observables en los grupos de aprendices que pertenecen a una misma lengua materna y que se derivan de las dificultades específicas que plantea la lengua extranjera que aprenden.

Puesto que nuestro estudio tiene un carácter sincrónico, los errores fosilizables, fosilizados y colecctivos son los que nos despiertan mayor interés y los que podremos invertigar con mayor detalle.

\section{Resultados y conclusiones}

Como comentado en la introducción, la presente investigación aún está en fase de desarrollo. Por ende, todavia es temprano para presentar resultados y discutirlos. No obstante, se espera que éstos puedan arrollar alguna luz sobre el proceso de aprendizaje y con ello podamos tener una idea más ajustada de como abordar ciertos contenidos que puedan llevar al desarrollo de errores fosilizables, facilitados por la transferencia negativa, tan recurrente en los aprendices adultos lusohablantes de español como lengua extranjera.

\section{Bibliografía}

ALMEIDA FILHO, J. C. P. (1995). Português para estrangeiros - interface como espanhol, Pontes Editores, Campinas.

CORDER, S. P. (1981). Error analysis and interlanguage, Oxford University Press, Oxford.

ELLIS, R. (1994). The study of second language acquisition, Oxford Applied Linguistics, 23, 824.

FERNÁNDEZ LÓPEZ, S. (1995). «Errores e interlengua en el aprendizaje del español como lengua extranjera», Didáctica. Lengua Y Literatura., 7, 203215. 
KeLLERMAN, E. (1995). «Crosslinguistic influence: Transfer to nowhere?» Annual Review of Applied Linguistics, 15, 125-150.

TARONE, E. (1992). "La variabilidad de la interlengua», en LICERAS, J. (Ed.), La Adquisicion de las Lenguas Extranjeras, Visor, Madrid.

WEINREICH, U. (1953). Languages in contact. Findings and problems, Linguistic Circle, New York.

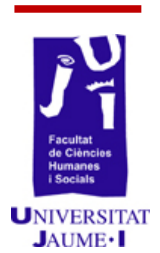

